OPEN ACCESS

Edited by:

Carl E. Stafstrom, Johns Hopkins Medicine,

United States

Reviewed by:

Ryan J. Felling,

Johns Hopkins University,

United States

Raul Chavez-Valdez,

Johns Hopkins Medicine,

United States

David Hsu,

University of Wisconsin-Madison,

United States

*Correspondence:

Divyen K. Shah

d.shah@qmul.ac.uk

†Joint senior authors.

Specialty section: This article was submitted to

Pediatric Neurology,

a section of the journal

Frontiers in Neurology

Received: 15 September 2017 Accepted: 06 February 2018

Published: 05 March 2018

Citation:

Shah DK, Ponnusamy V, Evanson J,

Kapellou O, Ekitzidou G, Gupta N, Clarke P, Michael-Titus AT and Yip PK (2018) Raised Plasma Neurofilament Light Protein Levels Are Associated

with Abnormal MRI Outcomes in Newborns Undergoing Therapeutic Hypothermia.

Front. Neurol. 9:86.

doi: 10.3389/fneur.2018.00086

\section{Raised Plasma Neurofilament Light Protein Levels Are Associated with Abnormal MRI Outcomes in Newborns Undergoing Therapeutic Hypothermia}

\author{
Divyen K. Shah ${ }^{1,2 *}$, Vennila Ponnusamy ${ }^{3,4}$, Jane Evanson ${ }^{1}$, Olga Kapellou ${ }^{5}$, \\ Georgia Ekitzidou ${ }^{5}$, Neelam Gupta ${ }^{6}$, Paul Clarke ${ }^{7}$, Adina T. Michael-Titus ${ }^{1+}$ \\ and Ping K. Yip ${ }^{1+}$
}

\begin{abstract}
${ }^{1}$ The Royal London Hospital, Barts Health NHS Trust, London, United Kingdom, ${ }^{2}$ The Centre for Neuroscience and Trauma, Barts and The London School of Medicine and Dentistry, Blizard Institute, Queen Mary University of London, London, United Kingdom, ${ }^{3}$ Centre for Genomics and Child Health, Barts and The London School of Medicine and Dentistry, Blizard Institute, Queen Mary University of London, London, United Kingdom, ${ }^{4}$ Ashford and St. Peter's Hospitals NHS Foundation Trust, Chertsey, United Kingdom, ${ }^{5}$ Homerton University Hospital NHS Foundation Trust, London, United Kingdom, ${ }^{6}$ University Hospital Southampton, Southampton, United Kingdom, ${ }^{7}$ Norfolk and Norwich University Hospitals NHS Foundation Trust, Norwich, United Kingdom
\end{abstract}

Aims and hypothesis: Hypoxic-ischemic encephalopathy (HIE) remains an important cause of death and disability in newborns. Mild therapeutic hypothermia $(\mathrm{TH})$ is safe and effective; however, there are no tissue biomarkers available at the bedside to select babies for treatment. The aim of this study was to show that it is feasible to study plasma neurofilament light (NfL) levels from newborns and to evaluate their temporal course. Hypothesis: Raised plasma NFL protein levels from newborns who undergo TH after HIE are associated with abnormal MRI outcomes.

Methods: Between February 2014 and January 2016, term newborns with HIE treated with $\mathrm{TH}$ for $72 \mathrm{~h}$ had plasma samples taken at three time points: (i) after the infant had reached target temperature, (ii) prior to commencing rewarming, and (iii) after completing rewarming. Infants with mild HIE who did not receive TH had a single specimen taken. NfL protein was analyzed using an enzyme-linked immunosorbent assay.

Results: Twenty-six newborns with moderate-severe HIE treated with TH were studied. Half of these had cerebral MRI predictive of an unfavorable outcome. Plasma NfL levels were significantly higher in the TH group with unfavorable outcome (median age $18 \mathrm{~h}$ ) compared to levels from both the mild HIE group and TH group with favorable outcome $(F=25.83, p<0.0001)$. Newborns who had MRls predictive of unfavorable outcome had significantly higher NfL levels compared to those with favorable outcomes, at all three time points (mixed models, $F=27.63, p<0.001$ ). A cutoff NfL level $>29 \mathrm{pg} / \mathrm{mL}$ at $24 \mathrm{~h}$ is predictive of an unfavorable outcome [sensitivity $77 \%$, specificity $69 \%$, positive predictive value (PPV) 67\%, negative predictive value (NPV) 72\%] with increasing predictive value until after rewarming (sensitivity 92\%, specificity 92\%, PPV 92\%, NPV 86\%). 
Interpretation of research: Plasma NfL protein levels may be a useful biomarker of unfavorable MRI outcomes in newborns with moderate-severe HIE and may assist in selecting newborns for adjunctive neuroprotective interventions. Larger studies with NfL testing at earlier time points are required.

Keywords: neurofilament proteins, hypoxic-ischemic encephalopathy, therapeutic hypothermia, MRI imaging, neuroprotection, biomarkers

\section{INTRODUCTION}

Perinatal asphyxia in the newborn remains an important cause of death and of disability in survivors. The resulting brain dysfunction and neonatal encephalopathy occurs in up to 4 per 1,000 live births in western countries $(1,2)$.

Mild therapeutic hypothermia (TH) treatment started within $6 \mathrm{~h}$ after birth, has been shown to be safe and effective in reducing death and disability, with numbers needed to treat (NNT) of 7-9 $(3,4)$. However, many babies go on to have adverse outcomes despite TH $(5,6)$. At present, empirical criteria are used to select babies for TH (7). Various compounds have been studied in the plasma, urine, and cerebrospinal fluid (CSF) as potential early biomarkers of CNS injury (8). There are no established blood biomarkers available to assist early cot side identification of babies most likely to benefit from neuroprotection. As a result, a number of babies with hypoxic-ischemic encephalopathy (HIE) who do not fulfill empirical criteria and who do not receive $\mathrm{TH}$ are noted to have adverse outcomes (9).

Neurofilaments, a group of intermediate sized filamentous proteins, are the most abundant cytoskeletal component, found predominantly in the myelinated axons of the central nervous system (10). They contribute to axonal volume and the radial diameter and hence are important for efficient transmission of action potentials. The neurofilament light (NfL) protein subunit is primarily expressed in large caliber myelinated axons.

When neurons are damaged, the NfL protein is released into the CSF and blood (10). An increased NfL protein level in CSF and blood is associated with the severity of neuropathology in adults with diseases including amyotrophic lateral sclerosis (ALS) (11), spinal cord injury (12), and multiple sclerosis (13). In asphyxiated newborns, neurofilament levels have been shown to be raised in CSF (14) and Toorell et al. (15) have recently reported raised levels in cord blood from asphyxiated newborns when compared to term controls. To date, there are no studies reporting the feasibility of studying plasma NfL levels in newborns with HIE who undergo TH and in the evaluation of their temporal course. We hypothesized that plasma NfL levels are significantly raised in newborns with cerebral MRI predictive of unfavorable neurodevelopmental outcomes.

If NfL is shown to be a reliable biomarker, then the selection of newborns for TH will be more effective with objective targeting of babies who will benefit most. The injury cascade affecting the newborn brain after hypoxia-ischemia continues for hours and days after the initial insult, with multiple mechanisms being implicated and hence various potential adjunctive neuroprotection targets could be considered (16). If reliable, NfL may prove to be a suitable biomarker of neuronal injury and useful for the evaluation of additional neuroprotective interventions.

\section{MATERIALS AND METHODS}

\section{Study Population}

Between January 2014 and January 2016, newborns were recruited into the Brain Injury Biomarkers in Newborns study from five tertiary neonatal centers; the Royal London Hospital, Homerton University Hospital, Ashford and St. Peter's Hospitals NHS Foundation Trust, University Hospital Southampton, and Norfolk and Norwich University Hospital. Babies were enrolled after written consent was obtained from the parents and this study had research ethics committee approval (REC reference: 13/LO/17380).

Two categories of babies were included: (1) 11 consecutively recruited term newborns admitted to the neonatal units with acidosis and/or mild HIE who did not fulfill standard criteria for $\mathrm{TH}$ and were managed conservatively and (2) babies who fulfilled standard cooling criteria (UK TOBY Cooling Register Clinician's Handbook) who underwent $\mathrm{TH}$ and had cerebral MR imaging. Of the latter group, the first 13 recruited with MR imaging predictive of favorable outcome and the first 13 with MR imaging predictive of unfavorable outcomes were included in the study. Exclusion criteria included death before an MRI was obtained (TH group), images degraded by significant motion artifact, major congenital anomaly, or a primary diagnosis of an inborn error of metabolism.

Blood from the babies with mild HIE who did not undergo $\mathrm{TH}$ was obtained at a single time point. Samples from babies who received $\mathrm{TH}$ were obtained at three time points in the first week after birth: (i) after the target temperature of $33-34^{\circ} \mathrm{C}$ was reached (S1), (ii) prior to rewarming (S2), and (iii) after rewarming was completed (S3).

\section{Preparation and Analysis of Plasma Samples}

Collected blood samples were transferred into spray-coated $\mathrm{K}_{2}$ EDTA tubes (Fisher Scientific Ltd., Loughborough, UK), then centrifuged at $15,000 \times g$ for 10 min to separate plasma, which was stored at $-80^{\circ} \mathrm{C}$ until analysis.

A commercially available enzyme-linked immunosorbent assay (ELISA) (UD51001, IBL-International GmbH) was used to quantify NfL. Bovine NfL standards ranging from 0 to $10,000 \mathrm{pg} / \mathrm{mL}$ and $100 \mu \mathrm{L}$ of diluted samples from each newborn $(50 \mu \mathrm{L}$ of neat plasma sample diluted with $160 \mu \mathrm{L}$ sample diluent) were run in duplicate, following the manufacturer's instructions. The optical density of each sample was determined at a wavelength of $450 \mathrm{~nm}$. NfL concentrations were calculated using the inverse formula: $x=c(((a-d) /$ 
$(y-d))-1)^{1 / b}$ provided by the online ELISA software (http:// elisaanalysis.com). This study was carried out with three NfL ELISA plates from different batches at different times, to ensure the test was not affected by variability between plates. Each ELISA plate contained samples from all three groups and from different time points. Each plate was processed at a different time. The mean of the two results was reported. A coefficient of variation of up to $10 \%$ was accepted.

\section{Outcomes}

Infants who underwent $\mathrm{TH}$ had cerebral MR imaging. MRI was performed at local centers with conventional T1-weighted and T2-weighted sequences at 1.5 T. MR images were independently rated by a neuroradiologist (JE) and a neonatologist with imaging expertise (OK) who were blinded to clinical information. All images were of sufficient quality to be reportable by each rater. Patterns of MRI injury were classified into two groups using the system described by Rutherford et al. (17), which has prognostic value in infants who have undergone $\mathrm{TH}$. Infants with an unfavorable outcome had a severe pattern of injury including reversed or abnormal signal intensity bilaterally on T1- and/or T2-weighted sequences in the posterior limb of the internal capsule (PLIC); multifocal or widespread abnormal signal intensity in the basal ganglia and thalami (BGT); and severe widespread white matter (WM) lesions including infarction, hemorrhage and long T1 and T2. Infants with MRIs predictive of a favorable outcome had either normal images or less severe patterns of injury that are associated with normal or only mildly abnormal neurodevelopmental outcomes. Consensus was reached in cases of disagreement.

Infants who did not qualify for $\mathrm{TH}$ were classified as having a favorable outcome if they had a normal neurologic examination at $24 \mathrm{~h}$ of age and were discharged home on suck feeding. These infants did not have MR imaging.

\section{Statistical Analysis}

Statistical analysis was carried out using SPSS (version 22). Continuous variables between groups were compared using ANOVA and proportions were compared using Pearson's chisquare statistic. NfL levels for S1 were positively skewed and were log transformed prior to analysis with ANOVA. Receiver operated characteristics (ROC) curves were created comparing the mild HIE group with the TH unfavorable outcome group at $\mathrm{S} 1$ and also comparing the $\mathrm{TH}$ favorable with the unfavorable outcome group at all three time points S1, S2, and S3. For each ROC curve, a cutoff level was obtained where the sensitivity + specificity was at its maximum. All graphs and ROC were created using GraphPad Prism (version 6.0). $p<0.05$ was used as the level of statistical significance.

\section{RESULTS}

The perinatal characteristics of the newborns studied are described in Table 1. Babies in the unfavorable outcome group were more likely to have had a lower 10-min Apgar score, and to have had chest compressions at resuscitation, anticonvulsants, and inotropic support.

\section{MRI Outcome}

The 26 babies who underwent $\mathrm{TH}$ all had MRI performed at a median age of 9 [interquartile range (IQR) 8,11] days. There was no statistical difference in age at MRI between the 13 babies with MRI predictive of favorable outcome and the other 13 with MRI predictive of unfavorable outcome $(F=0.89, p=0.56)$ (Table 1). Of the 13 infants with images predictive of unfavorable outcome, six had

TABLE 1 | Comparison of the clinical characteristics of the three groups of babies studied.

\begin{tabular}{|c|c|c|c|c|}
\hline & $\begin{array}{l}\text { Mild } \\
\text { No therapeutic hypothermia (TH) }\end{array}$ & $\begin{array}{l}\text { Mod-severe } \\
\text { TH favorable }\end{array}$ & $\begin{array}{l}\text { Mod-severe } \\
\text { TH unfavorable }\end{array}$ & $p$-Value \\
\hline$n$ & 11 & 13 & 13 & \\
\hline Male & $5 / 11$ & $9 / 13$ & $9 / 13$ & 0.39 \\
\hline Birth weight (g) & $3,360(2,971,3,987)$ & $3,546(3,045,3,880)$ & $3,440(2,800,3,770)$ & 0.70 \\
\hline PMA & $40(39,41.29)$ & $39.57(38.86,41)$ & $40.14(38,41)$ & 0.73 \\
\hline 10-min Apgar & $9(8,9)$ & $6(4,7)$ & $5(4,6)$ & 0.001 \\
\hline Chest compressions & $0 / 9$ & $3 / 10$ & $7 / 11$ & 0.035 \\
\hline Resuscitation cardiac drugs & $0 / 9$ & $2 / 12$ & $5 / 11$ & 0.13 \\
\hline Worst $\mathrm{pH}$ in first hour & $6.96(6.91,7.03)$ & $6.90(6.71,7.00)$ & $6.87(6.77,6.94)$ & 0.27 \\
\hline Worst base deficit in first hour & $-15(-12,-8)$ & $-17(-13,-21)$ & $-20(-18,-23)$ & 0.14 \\
\hline Maternal pyrexia & $2 / 10$ & $0 / 12$ & $0 / 12$ & 0.22 \\
\hline Chorioamnionitis & $0 / 9$ & $0 / 13$ & $0 / 12$ & 0.52 \\
\hline Sentinel event & $1 / 11$ & $4 / 13$ & $3 / 13$ & 0.25 \\
\hline Meconium aspiration & $0 / 10$ & $1 / 13$ & $2 / 12$ & 0.41 \\
\hline Blood glucose (<2.6 mmol/l) & $0 / 8$ & $0 / 8$ & $0 / 9$ & 0.83 \\
\hline Positive blood culture & $0 / 10$ & $0 / 13$ & $1 / 13\left(\mathrm{CONS}^{\star}\right)$ & 0.38 \\
\hline Clinical seizures & $0 / 10$ & $8 / 13$ & $12 / 13$ & $<0.001$ \\
\hline Anticonvulsants given & $0 / 10$ & $7 / 13$ & $10 / 12$ & $<0.001$ \\
\hline Inotropes used & $0 / 11$ & $2 / 13$ & $9 / 12$ & $<0.001$ \\
\hline Age at MRI scan (days) & - & $8(7,9)$ & $9(8,12)$ & 0.56 \\
\hline
\end{tabular}

Data in brackets represent median interquartile range.

CONS*, coagulase negative staphylococci. 
TABLE 2 | Patients arranged in descending rank order of neurofilament light levels at S3 in relation to MRI findings and neurological exam at discharge. The latter is classified primarily in terms of abnormality of tone.

\begin{tabular}{|c|c|c|c|c|c|c|c|c|c|c|c|c|c|}
\hline & $\begin{array}{c}\mathrm{S1} \\
(\mathrm{pg} / \mathrm{mL})\end{array}$ & $\begin{array}{c}\mathrm{S} 2 \\
(\mathrm{pg} / \mathrm{mL})\end{array}$ & $\begin{array}{c}\text { S3 } \\
(\mathrm{pg} / \mathrm{mL})\end{array}$ & $\begin{array}{c}\text { Age at } \\
\text { peak (h) }\end{array}$ & $\begin{array}{c}\text { Electrical } \\
\text { seizures } \\
(\mathrm{Y} / \mathrm{N})\end{array}$ & PLIC & BGT & WM & Cortex & $\begin{array}{l}\text { Composite } \\
\text { MRI Group }\end{array}$ & $\begin{array}{c}\text { Sentinel } \\
\text { event } \\
\text { (Y/N) }\end{array}$ & Comment & $\begin{array}{l}\text { Neurologic } \\
\text { exam prior to } \\
\text { discharge }\end{array}$ \\
\hline 1 & 2,506 & 2,255 & 4,698 & 100 & $Y$ & Loss & Severe & Moderate & Normal & U & $\mathrm{N}$ & & Severe, died \\
\hline 2 & 1,999 & 1,615 & 1,993 & 18 & $\mathrm{~N}$ & Equivocal & Normal & Severe & Moderate & $U$ & $Y$ & $\begin{array}{l}\text { Cord } \\
\text { prolapse }\end{array}$ & Mild \\
\hline 3 & 0 & 189 & 1,626 & 124 & $Y$ & Loss & Severe & Severe & Severe & $U$ & $\mathrm{~N}$ & & Severe, died \\
\hline 4 & 70 & 423 & 1,014 & 114 & $N$ & Loss & Severe & Mild & Mild & $U$ & Y & $\begin{array}{l}\text { Breech, head } \\
\text { entrapment }\end{array}$ & $\begin{array}{l}\text { Moderate, } \\
\text { tube feeds }\end{array}$ \\
\hline 5 & 1,046 & 1,512 & 874 & 31 & Y & Normal & Moderate & Normal & Normal & $U$ & $\mathrm{~N}$ & & Normal \\
\hline 6 & 516 & $\mathrm{n} / \mathrm{a}$ & 849 & 96 & $N$ & Equivocal & Normal & Severe & Severe & $U$ & Y & $\begin{array}{l}\text { Placental } \\
\text { abruption }\end{array}$ & $\begin{array}{l}\text { Severe, } \\
\text { tube feeds }\end{array}$ \\
\hline 7 & 413 & 381 & 844 & 118 & Y & Normal & Moderate & Severe & Moderate & $U$ & $\mathrm{~N}$ & & $\begin{array}{l}\text { Moderate, } \\
\text { tube feeds }\end{array}$ \\
\hline 8 & 29 & 225 & 674 & 95 & $\mathrm{~N}$ & Loss & Severe & Severe & Severe & $U$ & Y & $\begin{array}{l}\text { Shoulder } \\
\text { dystocia }\end{array}$ & $\begin{array}{l}\text { Severe, } \\
\text { tube feeds }\end{array}$ \\
\hline 9 & 0 & $\mathrm{n} / \mathrm{a}$ & 563 & 94 & Y & Equivocal & Moderate & Mild & Moderate & $U$ & $\mathrm{~N}$ & & $\begin{array}{l}\text { Moderate, } \\
\text { tube feeds }\end{array}$ \\
\hline 10 & 0 & 0 & 511 & 96 & $\mathrm{n} / \mathrm{a}$ & Loss & Moderate & Mild & Normal & $U$ & $\mathrm{~N}$ & & $\begin{array}{l}\text { Severe, } \\
\text { tube feeds }\end{array}$ \\
\hline 11 & 0 & 294 & 506 & 100 & $Y$ & Normal & Mild & Mild & Normal & $\mathrm{F}$ & $\mathrm{N}$ & & Normal \\
\hline 12 & 681 & 419 & 490 & 18 & $N$ & Loss & Severe & Normal & Moderate & $\cup$ & $\mathrm{N}$ & & Normal \\
\hline 13 & 141 & 321 & 403 & 115 & $\mathrm{~N}$ & Normal & Normal & Normal & Normal & $\mathrm{F}$ & Y & $\begin{array}{l}\text { Placental } \\
\text { abruption }\end{array}$ & Normal \\
\hline 14 & 99 & 577 & 381 & 67 & $\mathrm{~N}$ & Normal & Normal & Normal & Normal & $\mathrm{F}$ & $Y$ & $\begin{array}{l}\text { Head } \\
\text { entrapment }\end{array}$ & Normal \\
\hline 15 & 28 & 77 & 336 & 101 & $\mathrm{~N}$ & Normal & Normal & Mild & Mild & $\mathrm{F}$ & Y & $\begin{array}{l}\text { Shoulder } \\
\text { dystocia }\end{array}$ & Normal \\
\hline 16 & 173 & 159 & 334 & 101 & $\mathrm{~N}$ & Loss & Severe & Moderate & Mild & $U$ & $\mathrm{~N}$ & & Normal \\
\hline 17 & 277 & 457 & 324 & 62 & $\mathrm{~N}$ & Normal & Normal & Normal & Normal & $\mathrm{F}$ & $\mathrm{N}$ & & Mild \\
\hline 18 & 166 & 160 & 242 & 86 & $Y$ & Normal & Mild & Normal & Normal & $\mathrm{F}$ & $\mathrm{N}$ & & Normal \\
\hline 19 & 0 & 71 & 164 & 108 & $\mathrm{n} / \mathrm{a}$ & Normal & Normal & Mild & Normal & $\mathrm{F}$ & $\mathrm{N}$ & & Normal \\
\hline 20 & 148 & 172 & 151 & 48 & Y & Loss & Moderate & Severe & Normal & $U$ & $\mathrm{~N}$ & & $\begin{array}{l}\text { Moderate, } \\
\text { tube feeds }\end{array}$ \\
\hline 21 & 0 & 41 & 143 & 115 & $\mathrm{n} / \mathrm{a}$ & Normal & Normal & Normal & Normal & $\mathrm{F}$ & $\mathrm{N}$ & & Normal \\
\hline 22 & 0 & 0 & 136 & 114 & Y & Normal & Normal & Moderate & Normal & $\mathrm{F}$ & $\mathrm{N}$ & & Normal \\
\hline 23 & 0 & 0 & 59 & 102 & $\mathrm{~N}$ & Normal & Normal & Normal & Normal & $\mathrm{F}$ & $\mathrm{N}$ & & Normal \\
\hline 24 & 0 & 0 & 8 & - & $\mathrm{n} / \mathrm{a}$ & Normal & Normal & Normal & Normal & $\mathrm{F}$ & $\mathrm{N}$ & & Normal \\
\hline 25 & 0 & 0 & 0 & - & $Y$ & Normal & Normal & Mild & Mild & $\mathrm{F}$ & $\mathrm{N}$ & & Normal \\
\hline 26 & 0 & 0 & 0 & - & $\mathrm{N}$ & Normal & Normal & Normal & Normal & $\mathrm{F}$ & Y & $\begin{array}{l}\text { Uterine } \\
\text { rupture }\end{array}$ & Normal \\
\hline
\end{tabular}

PLIC, posterior limb of internal capsule; BGT, basal ganglia and thalami; WM, white matter; $n / a$, sample was not available; $F$, favorable; $U$, unfavorable; $y$, yes; $n$, no.

a combination of subcortical WM and BGT abnormalities, five had predominantly BGT abnormalities, and two had predominantly subcortical WM abnormalities (Table 2). Representative images are shown in Figure 1. Overall scores for the MR images of the 26 babies who underwent $\mathrm{TH}$ showed $96 \%$ inter-rater agreement.

\section{Perinatal Characteristics and NfL Levels}

Median (IQR) age at obtaining the first sample in the mild HIE group was $24(13,34) \mathrm{h}$ (Table 3$)$. Three of the 11 babies in this group had a detectable or raised NfL level. Median (IQR) NfL levels in this whole group were $0(0,41) \mathrm{pg} / \mathrm{mL}$. In the babies who had received TH $(n=26)$, there was no statistically significant correlation between the highest NfL level and 10-min Apgar, worst $\mathrm{pH}$, and base deficit in the first hour. Similarly, there was no significant correlation between the presence of electrographic seizures on amplitude-integrated EEG monitoring and NfL levels.

\section{Plasma NfL Levels in Babies with Unfavorable Outcome Compared to the Other Groups}

There was no significant difference between groups in age at obtaining samples S1, S2, and S3 (Table 3). Median values (IQR) of the three groups at the different time points are shown in Table 3. The evolution of NfL levels during cooling for individual babies is shown in Figure 2. After log transformation and analysis of variance, the difference in initial NfL levels between the three groups was highly significant $(F=25.83$, $p<0.0001)$. 


\section{Temporal Increase in Plasma NfL Level in Babies Treated with TH}

For the babies who underwent TH, sequential NfL levels were higher in those with MR-evident brain injury predictive of unfavorable outcome compared to NfL levels in those with MRI predictive of favorable outcomes (mixed models, two-way ANOVA, $F=27.6, p<0.001$ ) (Figure 3). In both groups, median NfL levels increased over the course of TH treatment; initial NfL levels (S1) were not only higher in babies with unfavorable outcomes but

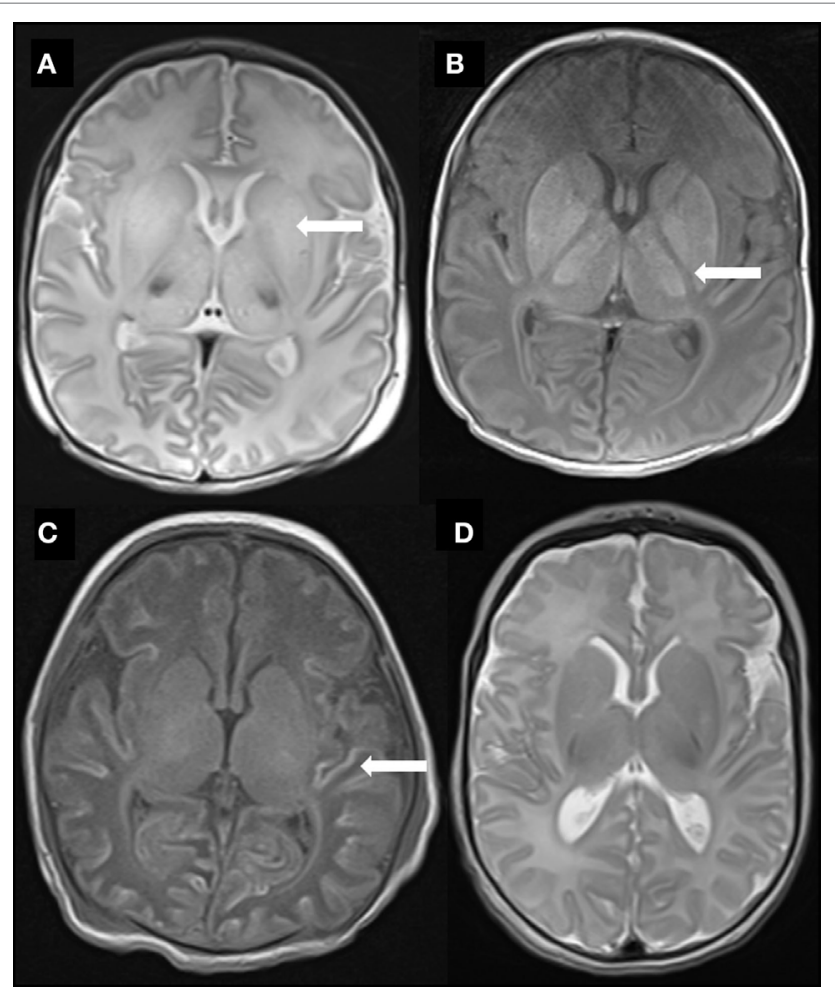

FIGURE 1 | Representative MR images of study babies. (A) Diffuse high T2 signal throughout the basal ganglia (arrow) from baby 3. (B) Abnormal high $\mathrm{T} 1$ signal throughout the basal ganglia and thalami as well as complete loss of signal in the posterior limb of internal capsule (arrow) also from baby 3. (C) Widespread T1 high signal (arrow) in cortex ("cortical highlighting") from baby

6. (D) Normal signal throughout the basal ganglia in T2-weighted image from baby 23 . also remained higher throughout the time course, in comparison to those from babies with favorable outcomes.

All infants with unfavorable outcomes had some rise in NfL levels. All infants with NfL levels $>400 \mathrm{pg} / \mathrm{mL}$ at the first sample $(n=6)$ had unfavorable outcomes. Of the 13 infants with favorable outcomes, 10 had some rise in NfL levels. Of these 10, four had completely normal MR imaging.

Six infants from the whole TH group with raised NfL levels in the first sample after reaching target hypothermia temperature (S1) showed a decrease in levels by the time of the second sample prior to commencing rewarming (S2). Yet all six showed a subsequent rise in NfL levels by the time of the third sample (post rewarming, S3).

\section{Diagnostic Accuracy of Plasma NfL Levels in Prediction of Unfavorable Outcome}

ROCs were performed to evaluate the diagnostic value of plasma NfL levels in predicting MRI predictive of abnormal outcome in newborns with HIE. At all three sampling time points, NfL cut-off levels likely to be predictive of unfavorable outcomes were obtained (Figure 4; Table 3). In babies undergoing $\mathrm{TH}$, an NfL level of $>29 \mathrm{pg} / \mathrm{ml}$ at $\mathrm{S} 1$ was predictive of unfavorable outcome, with area under the curve (AUC) of $0.78, p=0.017$, sensitivity $77 \%$, and specificity $69 \%$ (Table 4 ). For S3, an NfL level of $>417 \mathrm{pg} / \mathrm{mL}$ was strongly predictive of unfavorable outcome with AUC 0.97, $p<0.0001$, sensitivity $92 \%$, and specificity $92 \%$. On arranging the subjects in rank order of NfL levels at S3, it is notable that babies with higher NfL levels were more likely to have cerebral abnormalities on MRI (Table 2). However, one newborn with an NFL level $>417 \mathrm{pg} / \mathrm{mL}$ had MRI predictive of favorable outcome and two with NFL levels $<417 \mathrm{pg} / \mathrm{mL}$ had MRI predictive of unfavorable outcomes.

\section{DISCUSSION}

We demonstrate that it is feasible to study NfL levels in plasma from newborn babies. In newborns undergoing TH for HIE, NfL levels were raised as early as $24 \mathrm{~h}$ after birth in those who had cerebral MRI predictive of unfavorable neurodevelopmental outcome. NfL levels rose during the course of TH and were highest post rewarming. A cutoff NfL level $>417 \mathrm{pg} / \mathrm{mL}$ after rewarming was strongly predictive of unfavorable outcome. Thus, raised NfL levels may be an important biomarker of neuronal, and more specifically axonal damage, in this group of babies.

TABLE 3 | The median neurofilament light levels and age after birth of patients in the study.

\begin{tabular}{|c|c|c|c|c|c|c|}
\hline & $\mathrm{S} 1$ (pg/mL) & Age (h) & $\mathrm{S} 2$ (pg/mL) & Age (h) & S3 (pg/mL) & Age (h) \\
\hline $\begin{array}{l}\text { Mild } \\
\text { No TH }\end{array}$ & $0(0,41)$ & $24(13,34)$ & - & - & - & - \\
\hline $\begin{array}{l}\text { Mod-severe } \\
\text { TH favorable }\end{array}$ & $0(0,110)$ & $16(11,22)$ & $71(0,200)$ & $57(48,62)$ & $164(47,327)$ & $103(96,108)$ \\
\hline $\begin{array}{l}\text { Mod-severe } \\
\text { TH unfavorable }\end{array}$ & $173(29,681)$ & $18(15,24)$ & $381(180,968)$ & $54(42,60)$ & $844(511,1,014)$ & $96(95,104)$ \\
\hline
\end{tabular}

Interquartile ranges are expressed in brackets.

$\mathrm{TH}$, therapeutic hypothermia. 


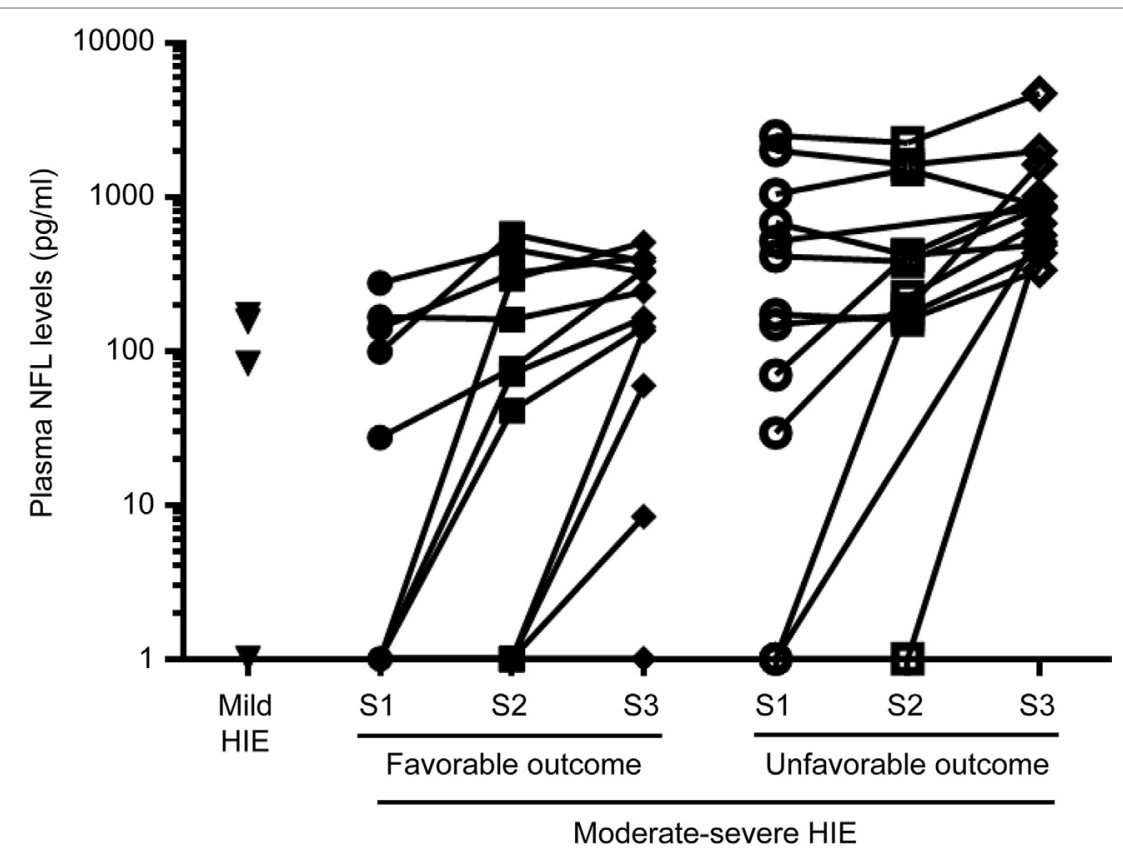

FIGURE 2 | Evolution of neurofilament light (NfL) over the three time points in the therapeutic hypothermia groups.

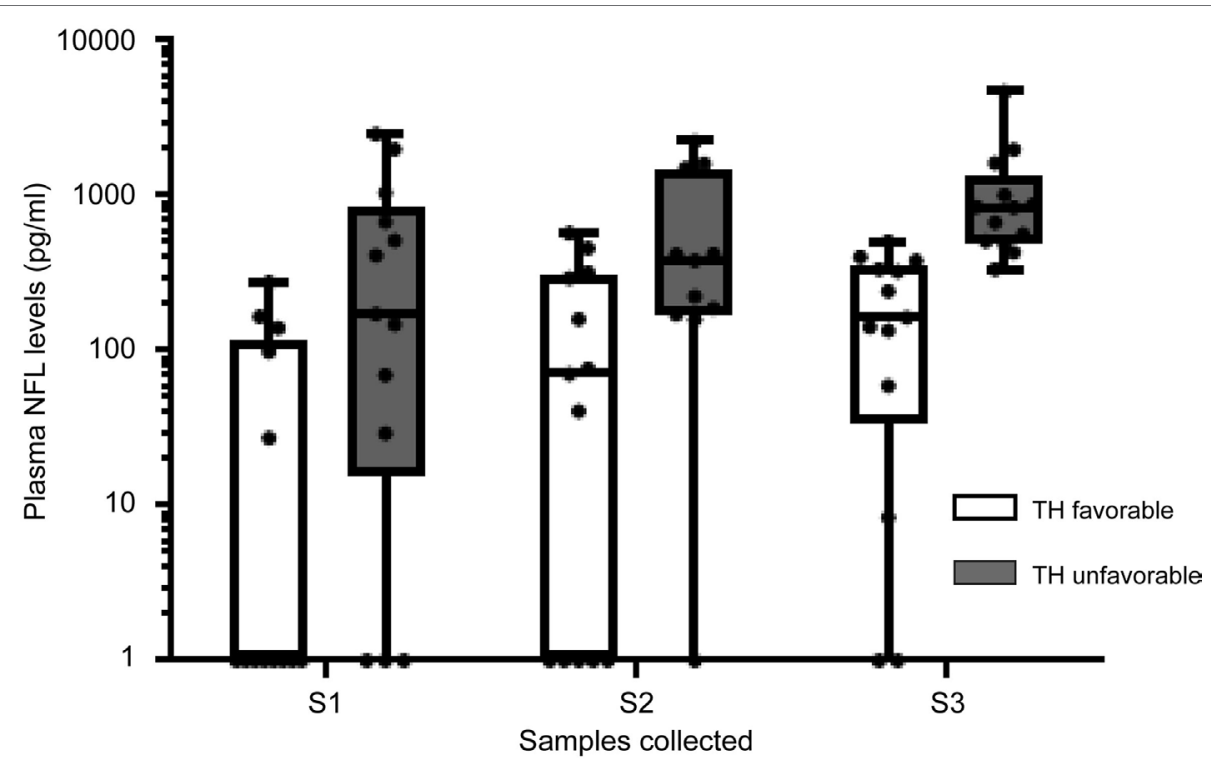

FIGURE 3 | Temporal changes in plasma neurofilament light (NfL) levels in groups with different severity of brain injury. Data expressed as median with interquartile ranges and whiskers with minimum to maximum.

Although safe, TH does not benefit all babies with $\operatorname{HIE}(3,4)$. Long-term follow-up from randomized controlled trials shows that up to $50 \%$ of babies who underwent TH nevertheless had adverse outcomes of death and disability $(5,6)$. At present, there are no clinically established biomarkers of brain injury available at the cot side that allow objective selection of babies for existing neuroprotective strategies such as $\mathrm{TH}$ or for stratifying babies for additional, novel interventions. The data from our study are consistent with the preliminary findings from Toorell et al. (15) and support plasma NfL as being a suitable candidate early biomarker worthy of further investigation. Furthermore, serum NfL levels have been shown to be raised in adult traumatic brain injury $(18,19)$.

In their study, Toorell et al. (15) compared NfL levels from the cord blood of 10 asphyxiated children with that of cord blood from 18 controls. Of their 10 cases, seven did not suffer 
A mild HIE vs Unfavorable outcome (S1)

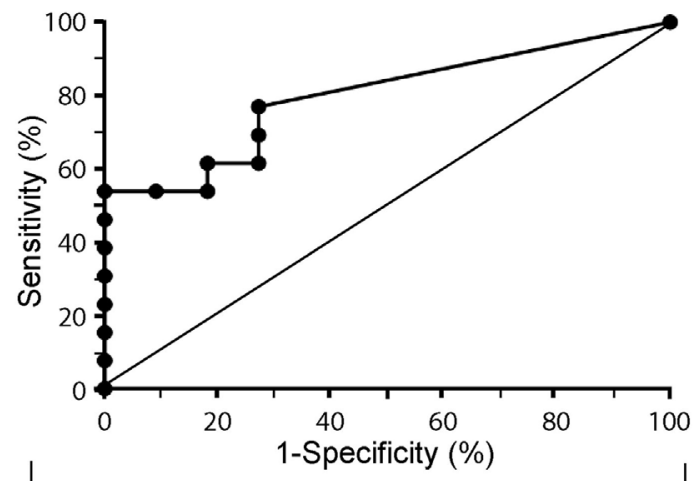

B Favorable vs Unfavorable outcome (S1)

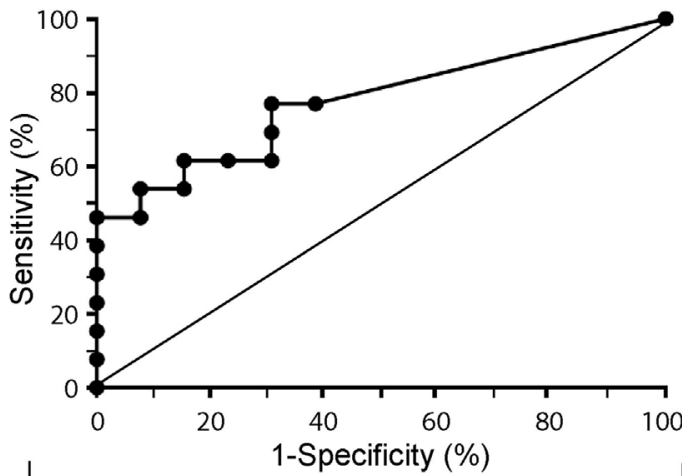

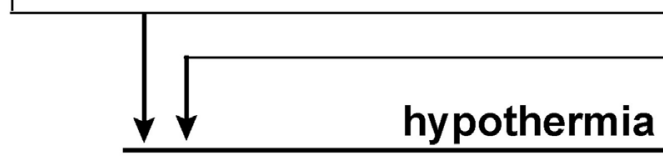

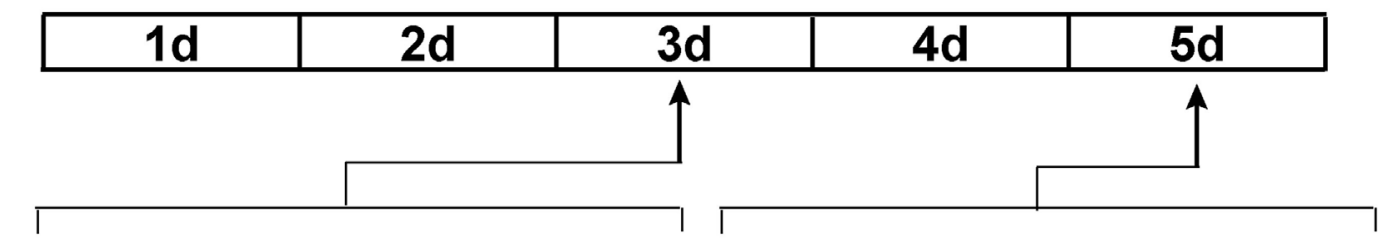

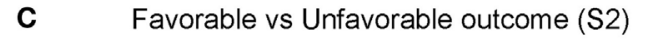

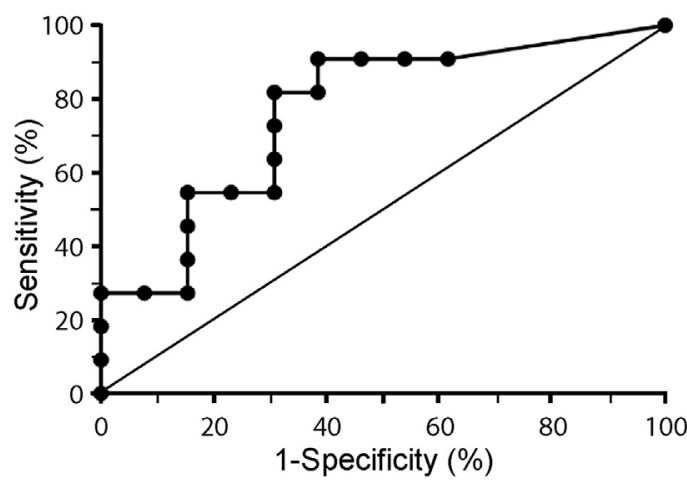

D

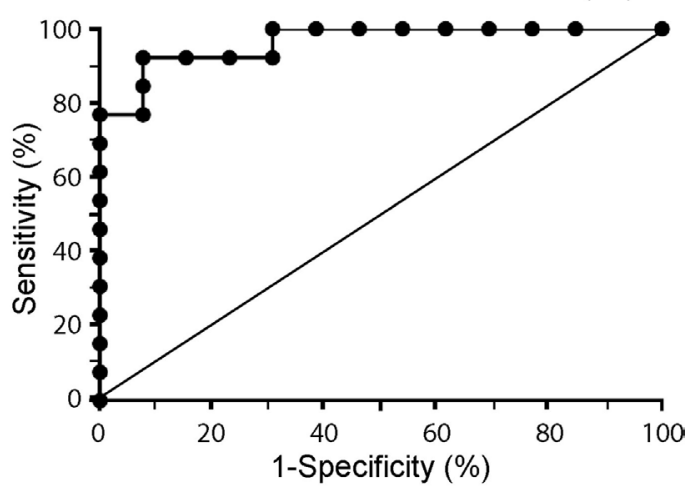

FIGURE 4 | Receiver operated characteristics curves of the neurofilament light (NfL) biomarker in the plasma of newborns with panel (A) representing a comparison of NfL level for babies with mild hypoxic-ischemic encephalopathy (HIE) [no therapeutic hypothermia (TH)] with TH babies with unfavorable outcome for the first sample and panels (B-D) representing a comparison of babies who had undergone TH at samples 1 (B), sample 2 (C), and sample 3 (D).

TABLE 4 | Diagnostic power of neurofilament light levels comparing babies newborns with favorable and unfavorable outcomes within the therapeutic hypothermia group at samples S1, S2, and S3.

\begin{tabular}{|c|c|c|c|c|c|c|c|c|}
\hline Sample & Area under curve & SE & $p$ Value & Cutoff (pg/ml) & Sensitivity (\%) & Specificity (\%) & PPV (\%) & NPV (\%) \\
\hline S1 & 0.775 & 0.094 & 0.017 & 29 & 77 & 69 & 67 & 73 \\
\hline S2 & 0.766 & 0.100 & 0.028 & 166 & 82 & 69 & 69 & 82 \\
\hline S3 & 0.965 & 0.032 & $<0.0001$ & 417 & 92 & 92 & 92 & 86 \\
\hline
\end{tabular}

PPV, positive predictive value; NPV, negative predictive value.

HIE. In our study, all cases undergoing TH had fulfilled local criteria for treatment. Although in this work we did not study cord blood, we were able to study the temporal evolution of the plasma NfL levels through the TH process and relate the NfL levels to MRI outcome. In this study, we did not carry out a comparison of a perinatal severity of "sickness" scoring system to NfL levels.

We noted that half the babies in the TH group had raised NfL levels in the first specimen. We speculate that the insult may have occurred some time prior to delivery in some of this group. In six 
of all the babies who received $\mathrm{TH}$ with raised NfL levels in the first specimen, there was a drop in NfL level noted in the second sample with the baby still being cooled. However, in all six, a rise in NfL levels was noted after rewarming was complete. This may be an observed effect of TH; a better understanding of the temporal changes in plasma NfL levels may also allow for more precise timing of the occurrence of the hypoxic-ischemic insult as well as better monitoring of the response to neuroprotective treatments.

To date, various blood-detected molecules have been proposed as potential biomarkers of brain injury after HIE (8). However, many of these markers are not specific to brain injury. One potential advantage of neurofilaments as biomarkers in the newborn is that they are neuron-specific cytoskeletal proteins, released into the CSF and blood when neurons are damaged (10). In this work, we have investigated only the NfL subunit because researchers from our institution and others have shown that NfL levels are raised in neurologic conditions in which neuronal and particularly axonal injury is important (11-13). Further work is required to test the utility of the other neurofilament subunits as potential biomarkers.

We used a commercially available sandwich ELISA kit that uses a heterophilic antibody designed for detecting NfL levels in the CSF, with a lower detection limit of $32 \mathrm{pg} / \mathrm{mL}$. At present, this test can be carried out and results obtained within $3 \mathrm{~h}$. We were able to show a significant difference between the groups in relation to cerebral injury on MRI, as well as a function of time. This antibody has been used in other studies on plasma and serum, albeit with other platforms (12). The newborns with unfavorable outcomes in this study had NfL levels (median $792 \mathrm{pg} / \mathrm{mL}$ ) that were higher than those observed in the plasma from adults with ALS (median $96 \mathrm{pg} / \mathrm{mL}$ ) (11). We postulate that this difference relates to the time course of the insult in newborns being more acute and the nature of the insult more global, compared to the more focal and chronic, slow-developing nature of the pathology in ALS.

A limitation of our study is that we did not have plasma specimens from cord blood, or from the babies before cooling was commenced. Also, we do not have samples from infants with moderate-severe HIE who did not receive TH. Although longer term neurodevelopmental outcomes are as yet unavailable, the method of scoring the MRIs that we used has been shown to be predictive of longer term outcomes (17).

\section{CONCLUSION}

Our data suggest that plasma NfL is a potentially useful biomarker for predicting unfavorable MRI outcomes in newborns with moderate-severe HIE undergoing TH. As a biomarker at $24 \mathrm{~h}$ of age and beyond, NfL levels may be of value for adjunctive neuroprotective treatments targeting the reperfusion and "repair" phases after hypoxia-ischemia. A larger study is required to confirm our findings, and should include plasma specimens from within the first $6 \mathrm{~h}$ after birth and explore the potential additional utility of intermediate and heavy subunits of neurofilaments. Further work is also required to correlate NfL levels with longer term neurodevelopmental outcomes.

\section{WHAT IS KNOWN ABOUT THIS TOPIC?}

- Therapeutic hypothermia does not benefit all newborns after hypoxic-ischemic encephalopathy

- There are no blood biomarkers that allow selection of babies for neuroprotection at present

- Neurofilament light (NfL) chains have been shown to be raised in blood and CSF from patients with other neurological pathology as well as cord blood from babies with asphyxia

\section{WHAT THIS STUDY ADDS}

- It is feasible to measure NfL levels, a marker of axonal injury, in the plasma of newborns

- NfL levels are raised at $24 \mathrm{~h}$ in newborns undergoing therapeutic hypothermia with cerebral tissue injury predictive of unfavorable outcome

- NfL levels may allow stratification of newborns to adjunctive brain saving treatments

\section{ETHICS STATEMENT}

This study was carried out in accordance with the recommendations of Bromley Research Ethics Committee with written informed consent from all parents. All parents gave written informed consent in accordance with the Declaration of Helsinki. The protocol was approved by the Bromley Research Ethics Committee.

\section{AUTHOR CONTRIBUTIONS}

DS conceptualized and designed the study, designed the data collection sheets, recruited patients for the study, performed the data analysis, drafted the initial manuscript, and approved the final manuscript as submitted. DS, the principal investigator, had full access to all the data in the study and took responsibility for the integrity of the data and the accuracy of the data analysis. VP assisted with designing the data collection sheets, recruited patients for the study, assisted with obtaining tissue samples and collecting and managing the data, assisted in drafting the manuscript, and approved the final manuscript as submitted. JE reviewed the MR images, assisted in preparing the manuscript, and approved the final manuscript. OK recruited patients for the study, reviewed the MR images, assisted in preparing the manuscript, and approved the final manuscript. GE recruited patients for the study, assisted with obtaining tissue samples and data collection, assisted in preparing the manuscript, and approved the final manuscript. NG recruited patients for the study, assisted with obtaining tissue samples and data collection, assisted in preparing the manuscript, and approved the final manuscript. PC assisted with designing the data collection sheets, recruited patients for the study, assisted with obtaining tissue samples and data collection, assisted in preparing the manuscript, and 
approved the final manuscript. AM-T provided intellectual input, assisted in preparing the manuscript and approved of the final manuscript. PY conceptualized and designed the study, performed the laboratory analysis, assisted with the data analysis, assisted in preparing the manuscript, and approved the final manuscript.

\section{ACKNOWLEDGMENTS}

We are grateful to the families who participated in this study and thank the medical and nursing staff on the neonatal units for their

\section{REFERENCES}

1. Badawi N, Kurinczuk JJ, Keogh JM, Alessandri LM, O'Sullivan F, Burton PR, et al. Antepartum risk factors for newborn encephalopathy: the Western Australian case-control study. BMJ (1998) 317(7172):1549-53. doi:10.1136/ bmj.317.7172.1554

2. Evans K, Rigby AS, Hamilton P, Titchiner N, Hall DM. The relationships between neonatal encephalopathy and cerebral palsy: a cohort study. J Obstet Gynaecol (2001) 21(2):114-20. doi:10.1080/01443610020025967

3. Edwards AD, Brocklehurst P, Gunn AJ, Halliday H, Juszczak E, Levene M, et al. Neurological outcomes at 18 months of age after moderate hypothermia for perinatal hypoxic ischaemic encephalopathy: synthesis and meta-analysis of trial data. BMJ (2010) 340:c363. doi:10.1136/bmj.c363

4. Jacobs SE, Berg M, Hunt R, Tarnow-Mordi WO, Inder TE, Davis PG. Cooling for newborns with hypoxic ischaemic encephalopathy. Cochrane Database Syst Rev (2013) 1:CD003311. doi:10.1002/14651858.CD003311.pub3

5. Azzopardi D, Strohm B, Marlow N, Brocklehurst P, Deierl A, Eddama O, et al. Effects of hypothermia for perinatal asphyxia on childhood outcomes. $N$ Engl J Med (2014) 371(2):140-9. doi:10.1056/NEJMoa1315788

6. Shankaran S, Pappas A, McDonald SA, Vohr BR, Hintz SR, Yolton K, et al. Childhood outcomes after hypothermia for neonatal encephalopathy. $N$ Engl $J$ Med (2012) 366(22):2085-92. doi:10.1056/NEJMoa1112066

7. Azzopardi D. UK TOBY Cooling Register Clinician's Handbook. 4th ed. (2010).

8. Douglas-Escobar M, Weiss MD. Biomarkers of hypoxic-ischemic encephalopathy in newborns. Front Neurol (2012) 3:144. doi:10.3389/fneur.2012. 00144

9. Murray DM, O'Connor CM, Ryan CA, Korotchikova I, Boylan GB. Early EEG grade and outcome at 5 years after mild neonatal hypoxic ischemic encephalopathy. Pediatrics (2016) 138(4):e20160659. doi:10.1542/peds.20160659

10. Petzold A. Neurofilament phosphoforms: surrogate markers for axonal injury, degeneration and loss. J Neurol Sci (2005) 233(1-2):183-98. doi:10.1016/j. jns.2005.03.015

11. Lu CH, Macdonald-Wallis C, Gray E, Pearce N, Petzold A, Norgren N, et al. Neurofilament light chain: a prognostic biomarker in amyotrophic lateral sclerosis.Neurology (2015) 84(22):2247-57. doi:10.1212/WNL.0000000000001642

12. Kuhle J, Gaiottino J, Leppert D, Petzold A, Bestwick JP, Malaspina A, et al. Serum neurofilament light chain is a biomarker of human spinal cord injury severity and outcome. J Neurol Neurosurg Psychiatry (2015) 86(3):273-9. doi:10.1136/jnnp-2013-307454 support. We are grateful to Ms Nicola Openshaw-Lawrence for assistance with managing the data.

\section{FUNDING}

We are grateful to the Barts Charity, London, UK and the National Institute for Health Research (NIHR) Brain Injury Healthcare Technology Co-operative based at Cambridge University Hospitals NHS Foundation Trust and University of Cambridge. The views expressed are those of the author(s) and not necessarily those of the NHS, the NIHR or the Department of Health.

13. Gaiottino J, Norgren N, Dobson R, Topping J, Nissim A, Malaspina A, et al. Increased neurofilament light chain blood levels in neurodegenerative neurological diseases. PLoS One (2013) 8(9):e75091. doi:10.1371/journal. pone.0075091

14. Blennow M, Sävman K, Ilves P, Thoresen M, Rosengren L. Brain-specific proteins in the cerebrospinal fluid of severely asphyxiated newborn infants. Acta Paediatr (2001) 90(10):1171-5. doi:10.1111/j.1651-2227.2001. tb03249.x

15. Toorell H, Zetterberg H, Blennow K, Sävman K, Hagberg H. Increase of neuronal injury markers Tau and neurofilament light proteins in umbilical blood after intrapartum asphyxia. J Matern Fetal Neonatal Med (2017):1-5. doi:10.1080/14767058.2017.1344964

16. Juul SE, Ferriero DM. Pharmacologic neuroprotective strategies in neonatal brain injury. Clin Perinatol (2014) 41(1):119-31. doi:10.1016/j.clp.2013.09.004

17. Rutherford M, Ramenghi LA, Edwards AD, Brocklehurst P, Halliday H, Levene $\mathrm{M}$, et al. Assessment of brain tissue injury after moderate hypothermia in neonates with hypoxic-ischaemic encephalopathy: a nested substudy of a randomised controlled trial. Lancet Neurol (2010) 9(1):39-45. doi:10.1016/ S1474-4422(09)70295-9

18. Shahim P, Zetterberg H, Blennow K. Neurofilament protein and antineurofilament antibodies following traumatic brain injury-reply. JAMA Neurol (2017) 74(3):363-4. doi:10.1001/jamaneurol.2016.5905

19. Al Nimer F, Thelin E, Nyström H, Dring AM, Svenningsson A, Piehl F, et al. Comparative assessment of the prognostic value of biomarkers in traumatic brain injury reveals an independent role for serum levels of neurofilament light. PLoS One (2015) 10(7):e0132177. doi:10.1371/journal.pone.0132177

Conflict of Interest Statement: The authors declare that the research was conducted in the absence of any commercial or financial relationships that could be construed as a potential conflict of interest.

The reviewer RC, RF and handling Editor declared their shared affiliation.

Copyright () 2018 Shah, Ponnusamy, Evanson, Kapellou, Ekitzidou, Gupta, Clarke, Michael-Titus and Yip. This is an open-access article distributed under the terms of the Creative Commons Attribution License (CC BY). The use, distribution or reproduction in other forums is permitted, provided the original author(s) and the copyright owner are credited and that the original publication in this journal is cited, in accordance with accepted academic practice. No use, distribution or reproduction is permitted which does not comply with these terms. 